\title{
Risk factors associated with short term mortality changes over time, after arrival to the emergency department
}

\author{
Camilla Nørgaard Bech ${ }^{*^{*}}$, Mikkel Brabrand ${ }^{2}$, Søren Mikkelsen ${ }^{3}$ and Annmarie Lassen ${ }^{4}$
}

\begin{abstract}
Background: Preventing death is the most important outcome pursued in the Emergency Department. Prompt accurate assessment, followed by competent and efficient investigation and treatment is the recipe sought. Abnormal physiological measurements are common antecedents to deterioration and therefore a cornerstone in many risk stratification tools. Some risk factors have their impact during the first few days after admittance, others have higher impact on 30 day mortality. Understanding the variance in impact of risk factors is relevant for future composition of risk stratification models.
\end{abstract}

Methods: We included patients aged 18 years or older, registered at the Emergency Department at Odense University Hospital from April 1st 2012 to September 30th 2014. We performed multivariate logistic regressions, adjusted for age, gender and comorbidity, to describe the relationship between potential risk factors and measures of short term mortality.

Results: A total of 43,178 were eligible for analysis. Median age was 56 (IQR 36-72) and 48.3\% were males. The over-all 30-day-mortality was 4\%. One third of deaths occurred within the first 2 days.

Higher age, male gender and comorbidity are all associated with immediate, 0-2 day, 3-7 day and 8-30 day mortality. The degree of acuteness at arrival defined by urgency-level, physician-assisted transfer to the Emergency Department and abnormal vital parameters are associated with 0-2 day mortality. High temperature at arrival shows no association in either mortality-group. Missing values are associated with immediate and 0-2 day mortality, but no association with mortality after 7 days.

Discussion: Abnormal vital parameters and degree of acuity at admission were strongly associated with mortality in the first hours and days after admission, where after the association decreased. The effect of other risk factors such as male gender, comorbidity and high age were time stable or even increasing over time..

Conclusions: The over-all 30-day mortality was $4 \%$. Physiology-related risk factors varied in strength of association throughout different mortality outcome measures.

Keywords: Emergency Department, Short term mortality, Risk factors, Observational cohort study

\footnotetext{
* Correspondence: Camilla.noergaard.bech@rsyd.dk

${ }^{1}$ Department of Emergency Medicine, Odense University Hospital, Sdr.

Boulevard 29, entrance 130, 1st floor, DK-5000 Odense C, Denmark

Full list of author information is available at the end of the article
} 


\section{Background}

Various early warning scores are used to identify patients, who are likely to deteriorate while hospitalized. Most of these warning scores are based on antecedents to deterioration such as abnormal vital values [1-6]. Other mortality-associated risk factors, such as lab results or time related factors are relevant in some - but not all settings, and the optimal method for risk assessment is often debated [7-17].

The impact on mortality of different risk factors might vary over time. Some risk factors are probably more important within the first few hours or days after arrival, while other risk factors have higher impact on 8-30 day mortality. Important risk factors related to death during the first few days, but not important for later death might be overlooked or blurred if timing of the outcome is not taken into account.

Since variation in impact of risk factor according to time after symptom occurrence (or arrival to the ED) is closely related to the efficacy of risk scores, our aim of the present study is to describe risk factors associated with mortality, distributed on four mortality-timespans after arrival to the ED; immediate death $(0-4 \mathrm{~h}), 4 \mathrm{~h}$ 2 days, 3-7 days and 8-30 days.

\section{Methods}

\section{Study design and setting}

This observational cohort study collected data on adults, who arrived to the ED at Odense University Hospital (OUH) during a $2 \frac{1}{2}$ year period. OUH is a tertiary hospital which also serves as a primary hospital for a population of 288,000 citizens. The ED provides $24-\mathrm{h}$ acute care with 105,000 visits per year (children and adults). The ED receives all acutely patients referred from either a primary care physician or patients who arrive by ambulance. The general admitting services for cardiology, hematology, oncology and obstetrics is placed elsewhere in-hospital.

The pre-hospital primary response is an ambulance manned with two emergency medical technicians or paramedics. The ambulance may be supplemented by a ground-based Mobile Emergency care Unit (MECU) manned with a specialist in anesthesiology [18]. In Denmark, hospital care is free of charge as part of the tax-funded healthcare system.

\section{Participants}

This study included all patients above 17 years, who arrived alive to the ED between April 1st 2012 and September 30th 2014. Patients with minor orthopedic injuries and patients without a Danish personal identification number were excluded. In order to avoid bias from repeated measurements, only first time ED-visit in the study period and the first set of recorded vital parameters were included for analysis.

\section{Data sources and variables}

The primary outcome was short term mortality, reported as immediate death (0-4 h after arrival), $4 \mathrm{~h}-2$ day mortality, 3-7 day and 8-30 day mortality. Arrival day were defined as day 0 .

Each patient-visit was linked to information from the two large population-based registries deriving from the unique Danish personal identification number: The Danish National Patient Registry and the Danish Civil Registration System [19, 20]. Collected data included patient demographics, with comorbidity defined by a condition recorded in a discharge diagnosis within 10 year prior to the index date, and described by Charlson Comorbidity Index [21]. Discharge diagnoses from the ED were collected and divided into major ICD-10 categories. We also included variables of day- or night-time and weekday or weekend. Mode of arrival was analyzed according to arrival at own accord, by ambulance or by ambulance assisted by MECU.

The first registered set of vital parameters; Glasgow coma score, heart rate, systolic blood pressure, temperature, peripheral oxygen saturation and respiratory rate were included. These data came primarily from electronic ED patient records, registered upon arrival to the ED. Secondly vital parameters from pre-hospital data registered in the MECU database or in ambulance records were used. Vital parameters were then divided into predefined strata, based on published guidelines [22].

The level of urgency was categorized according to the triage-assessment registered a arrival. The suspected degree of life or limb threat, assessment of vital parameters and the patient's presenting complaint are part of our triage system [1, 2] (see supplemental 1 for details on vital values). We defined the highest urgency-level as "very urgent", a patient-category with immediate assistance of a dedicated trauma- or medical emergency response team upon arrival to the ED. Our level 2 category of urgency was named "urgent" and was merged by the two middle triage-levels, 2 and 3, which consists of patients who needs to be seen by a physician within 15 or $60 \mathrm{~min}$. (Additional file 1).

\section{Statistical methods}

Demographic data are described as median, quartiles and range (where appropriate). Proportions are presented with $95 \%$ confidence intervals, based on binomial distribution. Patients were followed to death or 30 days following index date, whichever came first.

Logistic regressions determined the association between exposure-variables, distributed on four levels of short time mortality. Multivariate logistic regression, 
Table 1 Patient characteristics, time and urgency registrations

\begin{tabular}{|c|c|c|c|c|c|}
\hline & $\mathrm{n}$ & $\%$ & (Continued) & & \\
\hline Overall & 43.178 & 100 & & $\mathrm{n}$ & $\%$ \\
\hline Age, median (years (IQR)) & 56 & $(36-72)$ & Glascow Coma Score & & \\
\hline Gender & & & $14-15$ & 29.327 & 67.9 \\
\hline Female & 22.318 & 51.7 & $3-13$ & 905 & 2.1 \\
\hline Male & 20.861 & 48.3 & missing & 12,946 & 30.0 \\
\hline Charlson comorbidity Index & & & Temperature & & \\
\hline 0 & 25.962 & 60.1 & Normal & 31.817 & 73.7 \\
\hline 1 & 7.121 & 16.5 & $<35$ & 89 & 0.2 \\
\hline$\geq 2$ & 10.095 & 23.4 & $>39$ & 631 & 1.5 \\
\hline Mode of arriving & & & missing & 10,641 & 24.6 \\
\hline
\end{tabular}

Table 1 Patient characteristics, time and urgency registrations (Continued)
32.171

10.208

799

MECU

Time of arrival

8-15.59

$16-23.59$

00-6.59

Day of arrival

Mon-Thursday

Fri-Sunday

Urgency

Non-urgent

Urgent

Very urgent

missing

Vital parameters

Peripheral oxygen saturation (\%)

Normal
$<90 \%$
missing
Respiratory rate ( $\mathrm{rr} / \mathrm{min})$
Normal
$<5$
$>36$
missing

Systolic Blood Pressure $(\mathrm{mmHg})$

Normal

$<90$

missing

Heart rate (hr/min)

Normal

$<40$

$>140$

missing
21.461

15.262

6.455

25.089

18.089

11.367

25.063

1.833

4.915

33.359

858

8.961

31.471

30

351

11.326

36.024

477

6.677

36,218

99

284

6.587
74.5

23.6

1.9

49.7

35.4

14.9

58.1

41.9

26.3

58.0

4.3

11.4

77.3

2.0

20.7

72.9

0.1

0.8

26.2

83.4

1.1

15.5

83.9

0.2

0.6

15.3 adjusted for age group, gender and comorbidity was used to determine odds ratios (OR).

Missing values were handled as independent variables throughout the analysis.

All statistical analyses were performed with STATA software (V. 14.0 Stata Corporation LP, Texas, USA).

\section{Results}

A total of 182,032 contacts with an average of 199 contacts per day were initially registered. After excluding patients dead at arrival, and patients with minor orthopedic injuries, we included 43,178 individual adult patients with a first time contact to the ED. Median age was 56 (IQR 36-72) and 48.3\% were male.

Approximately three quarters of the cohort arrived at their own accord. The majority of arrivals occurred during daytime in weekdays and was evaluated as "urgent" at first assessment in the ED. Vital parameters were in general within predefined "normal" reference limits, with a proportion of missing values ranging from $15.5 \%$ in heart rate to $30.0 \%$ in GCS (Table 1 ).

The overall 30 day mortality was $4 \%$, with $0.7 \%$ dead within $4 \mathrm{~h}$ after arrival, $0.5 \%$ in $0-2$ day mortality, $0.9 \%$ in 3-7 day mortality and $1.9 \%$ dead in $8-30$ day mortality (Fig. 1). Conclusively, $30 \%$ of the patients who died within 30 days, died within the first 2 days after arrival to the ED.

Strong risk factors for immediate mortality were abnormal vital values as well as high urgency level at arrival. The strength of association with mortality of these risk factors declined considerable according to increasing time from arrival till death (Table 2).

In contrast, impact of comorbidity increased with time from arrival to death and age and male sex were time stable risk factors. Fever and time and day of arrival showed no association with mortality in any of the groups.

Missing values were associated with immediate and 02 day mortality but had no association after 7 days (Table 2). 
182,032 contacts of patients above 17 years to the Emergency Department of Odense University Hospital in the studyperiod

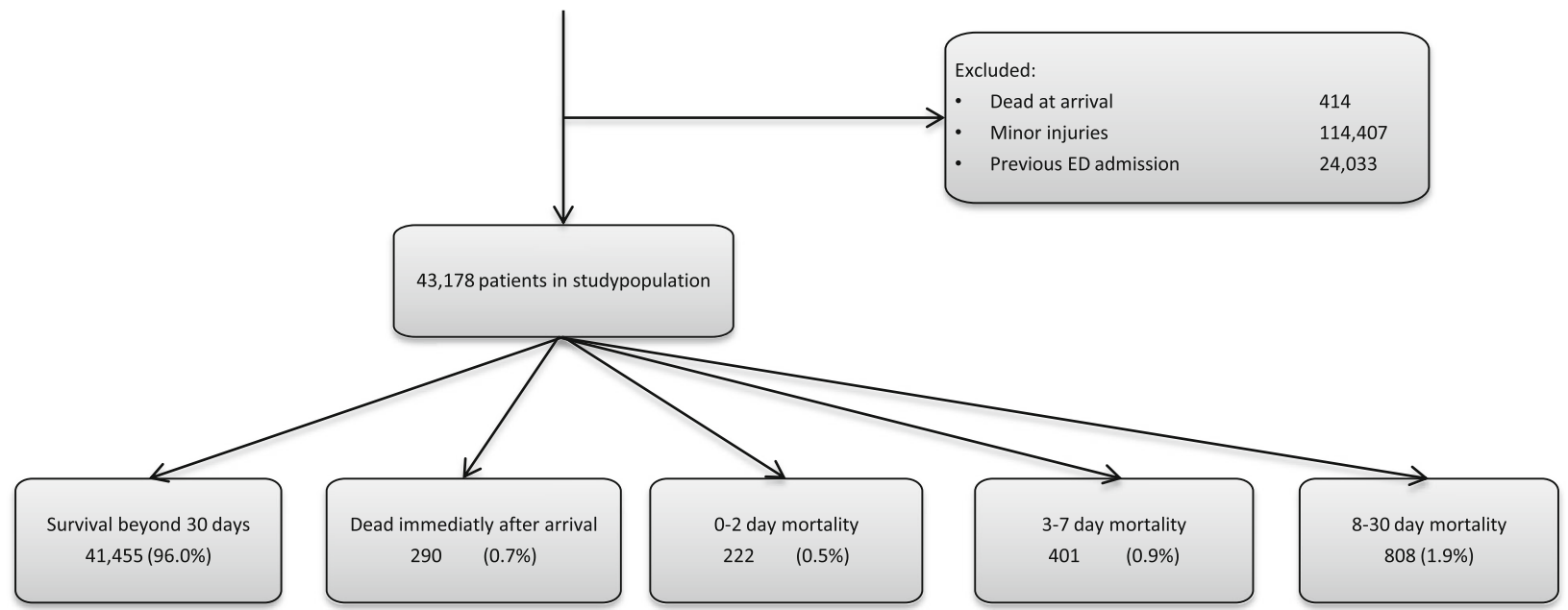

Fig. 1 Flowchart describing selection of cohort and distribution of outcome

Discharge diagnoses from the ED showed that a majority of patients, who died immediately after arrival, were diagnosed with diseases of the circulatory system. This was also the major discharge diagnose-category in the other mortality groups, except for 8-30 day mortality, where diagnoses of the respiratory system accounted for the majority. Diseases of the respiratory system was the second largest group of ICD diagnoses in both the $0-2$ day mortality and the 3-7 day mortality group. Injury, poisoning and external causes accounted for the second largest diagnose-category in the immediately dead group (Table 3).

\section{Discussion}

We showed an over-all-30 day mortality of $4 \%$. One third of the 30 day mortality occurred within 2 days after arrival to the ED. Abnormal vital signs are strong risk factors for mortality within the first 2 days and hereafter the association declines with time from arrival.

Our overall mortality is similar to previous results from other ED-settings [12, 23]. Also, abnormal vital parameters and the degree of acuity at arrival are also wellknown risk factors $[3,4]$.

Timing related to mortality as outcome is often not taken into consideration, when weighing the effect of risk factors. In our study, abnormal vital parameters and degree of acuity at admission are strongly associated with mortality in the first hours and days after admission, where after the association decreases. The effect of other risk factors such as male gender, comorbidity and high age are time stable or even increasing over time.
We excluded patients arriving at own accord with minor trauma, since these patients are only registered with a minimum of data and often not initially triaged. By doing this, we excluded a fraction of clinically stabile orthopedic patients, who were admitted directly in the orthopedic department for observation or surgery or discharged directly after examination. Many could have been assessed by a physician in the primary sector.

Patients who were not registered dead by a physician prior to ED arrival were included. Patients with major trauma or other seriously life-threatening clinical conditions, who were kept alive by active treatment from the medical staff at MECU, during transfer to the ED, are included. In cases, where active treatment ceased shortly after arrival to the ED and the patient died, they were included in the immediately dead group and add strength to the association with mortality in all parameters. In reality, active treatment is often ceased due to detailed knowledge on severe comorbidity, initial trauma scans or lab results which makes further treatment unethical.

A very large proportion of vital parameters and registration of urgency level were missing, particularly in the group of patients dying $0-4 \mathrm{~h}$ after arrival. This supports the well-known finding, that large amounts of missing data are present in trauma or acute-setting registries and is also a consequence of the use of secondary data sources where selection, quality and method of collection is not under the control of the researcher [24-26]. In our study, missing values in all variables are associated with $0-2$ day mortality, but the association 
Beck et al. Scandinavian Journal of Trauma, Resuscitation and Emergency Medicine (2018) 26:29

Page 5 of 9

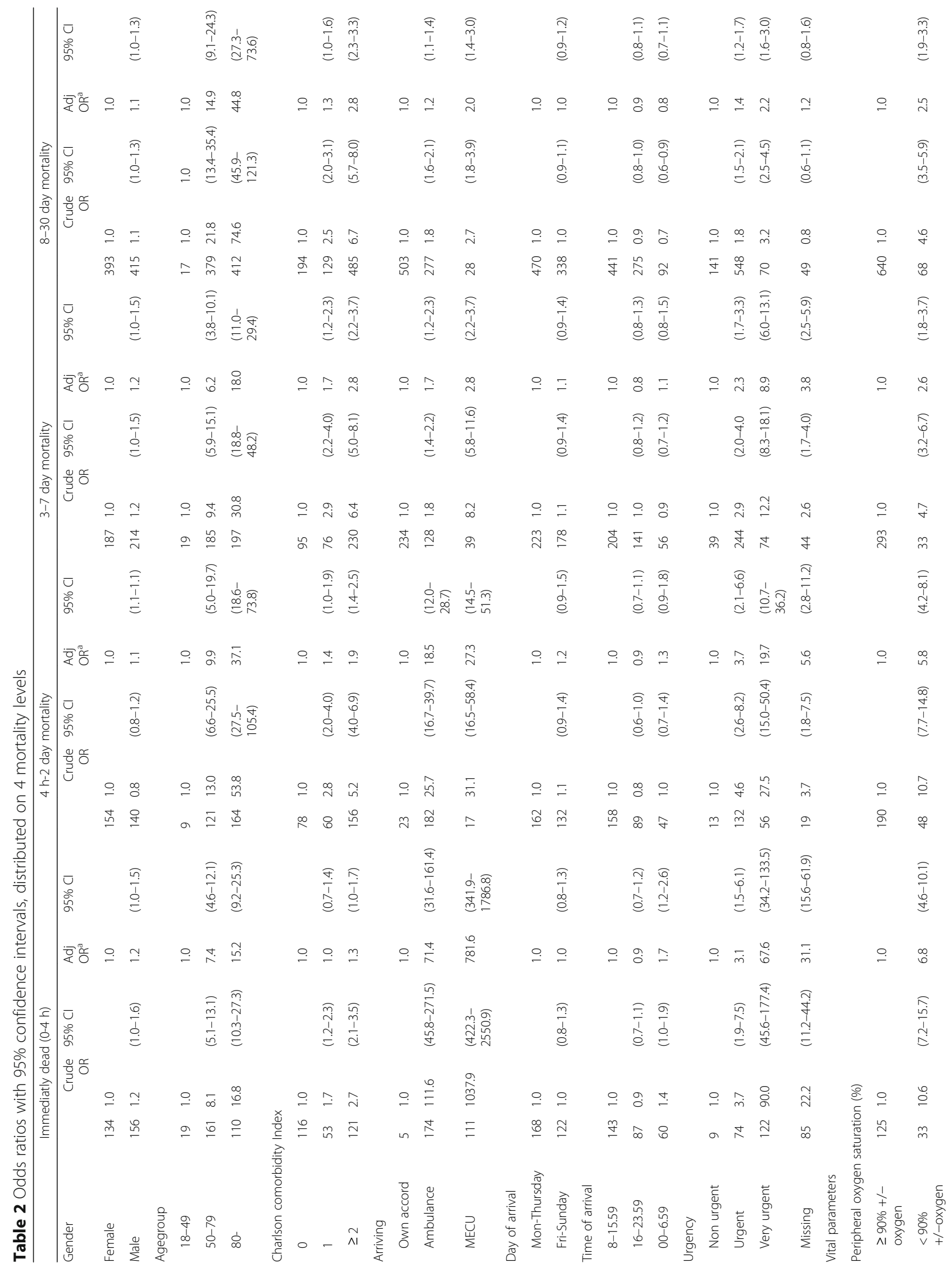




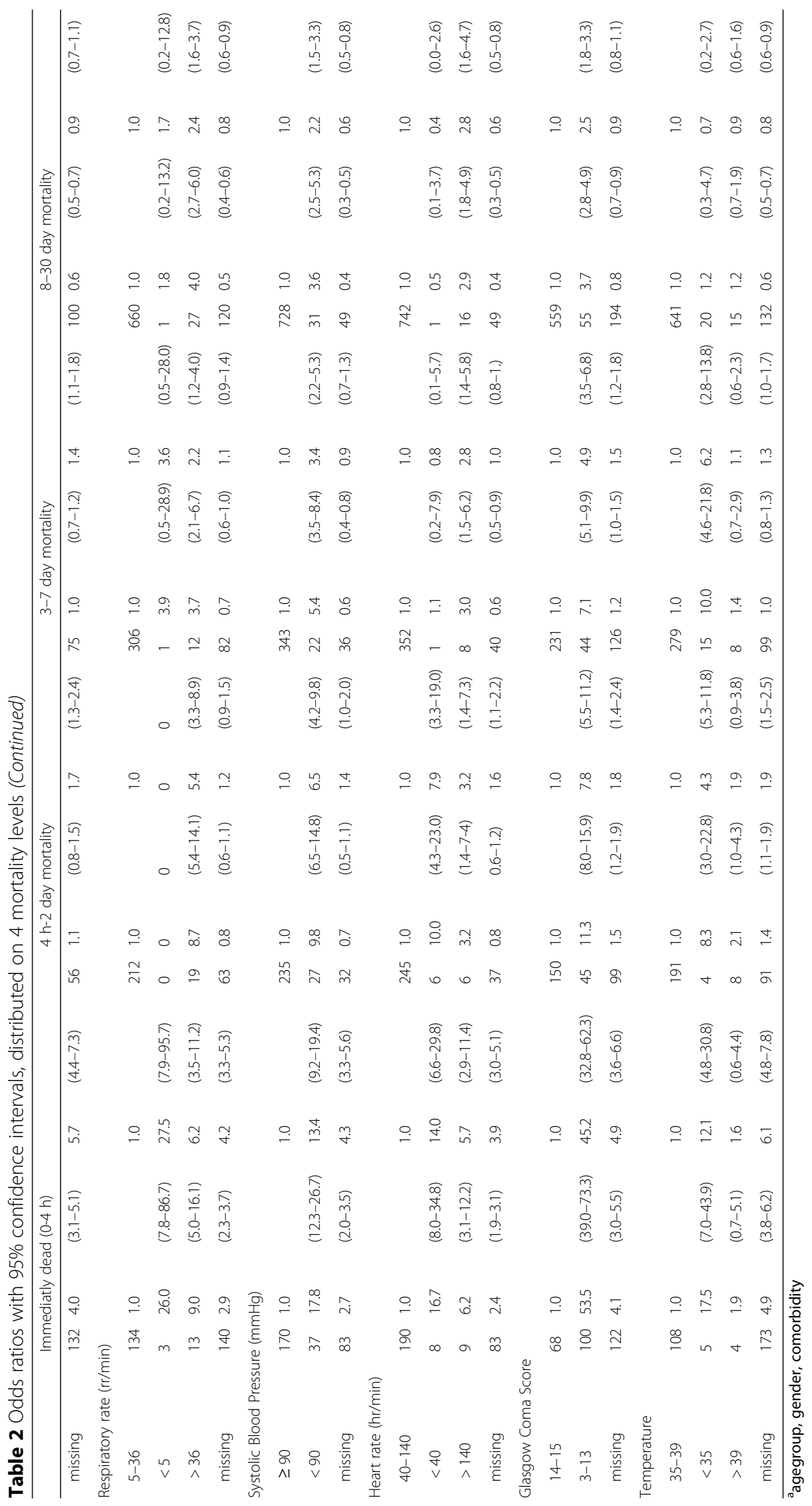


Table 3 Frequency discharge diagnosis according to major ICD-10groups, distributed on four mortality levels

\begin{tabular}{|c|c|c|c|c|c|c|c|c|c|c|c|}
\hline ICD-10 groups & Disease categories & Overall & & $0-4 \mathrm{~h}$ & & 4 h-2 day & & 3-7 day & & 8-30 day & \\
\hline & Total N (\%) & 1721 & $(100)$ & 290 & (100) & 222 & (100) & N 401 & (100) & N 808 & $(100)$ \\
\hline A00-B99 & $\begin{array}{l}\text { Certain infectious and } \\
\text { parasitic diseases }\end{array}$ & 107 & $(6.2)$ & 20 & (6.9) & 19 & (8.6) & 27 & $(6.7)$ & 41 & (5.1) \\
\hline C00-D89 & $\begin{array}{l}\text { Neoplasms and diseases } \\
\text { of blood and blodforming organs }\end{array}$ & 153 & (8.9) & 5 & $(1.7)$ & 12 & (5.4) & 33 & $(8.2)$ & 103 & $(12.7)$ \\
\hline E00-E90 & $\begin{array}{l}\text { Endocrine, nutritional } \\
\text { and metabolic diseases }\end{array}$ & 77 & $(4.5)$ & 5 & $(1.7)$ & 13 & (5.9) & 12 & (3.0) & 47 & (5.8) \\
\hline G00-G99 & Diseases of the nervous system & 17 & (1.0) & 2 & $(0.7)$ & 0 & 0 & 8 & (2.0) & 7 & $(0.9)$ \\
\hline $100-199$ & Diseases of the circulatory system & 454 & (26.4) & 159 & $(54.8)$ & 82 & (36.9) & 103 & $(25.7)$ & 110 & (13.6) \\
\hline J00-J99 & Disease of the respiratory system & 335 & $(19.5)$ & 27 & (9.3) & 39 & (17.6) & 86 & (21.4) & 183 & (22.6) \\
\hline K00-K93 & Diseases of the digestive system & 161 & (9.4) & 11 & $(3.8)$ & 25 & (11.3) & 43 & $(10.7)$ & 82 & $(10.1)$ \\
\hline N00-N99 & Disease of the genitourinary system & 54 & (3.1) & 2 & $(1.7)$ & 5 & $(2.3)$ & 10 & $(2.5)$ & 37 & (4.6) \\
\hline R00-R99 & $\begin{array}{l}\text { Symptoms, signs, and abnormal } \\
\text { clinical and labotory findings, } \\
\text { not elsewhere classified }\end{array}$ & 119 & (6.9) & 17 & $(5.9)$ & 15 & (6.8) & 26 & (6.5) & 61 & $(7.5)$ \\
\hline \multirow[t]{3}{*}{ S00-T98 } & $\begin{array}{l}\text { Injury, poisoning and certain } \\
\text { other conseqeunces } \\
\text { of external causes }\end{array}$ & 91 & (5.3) & 30 & (10.3) & 2 & (0.9) & 21 & (5.2) & 38 & (4.7) \\
\hline & $\begin{array}{l}\text { Others (mental, eye, ear, skin, } \\
\text { connective tissue, external causes etc) }\end{array}$ & 147 & (8.5) & 10 & (3.4) & 9 & (7.7) & 31 & (7.7) & 97 & (12.0) \\
\hline & Missing & 6 & (0.3) & 2 & $(0.7)$ & 1 & $(0.5)$ & 1 & $(0.2)$ & 2 & $(0.2)$ \\
\hline
\end{tabular}

disappears over time and missing data show no association after 7 days.

Our result reasserts the interpretation that registration and documentation in patient records is not always carried out due to logistics, prioritization, investigations and treatment in very acute situations, with very ill patients. One might also conclude that it can be fatal to miss vital parameter measurement and triage upon arrival to the ED if the patient is very sick, because lacking recognition of a serious clinical condition at arrival, can lead to dead within the first few days. Finally, the patient might be in such poor clinical condition at arrival, that the healthcare personnel can acknowledge the severity without measuring vital values.

The occurrence of missing data is a well-known premise of data from acute settings and in our studies, missing data in vital values and triage might represent immeasurable low or high values, values of zero, normal values observed but not registered, or simple lack of registration due to circumstances surrounding the patient at the time of assessment. The results in the multivariate analysis of this article reflect the fact that missing values are not missing at random, as OR related to missing data was high. If we were to omit these data, important information and future research aspects of risk factors associated with mortality in the ED would be lost. We do agree that missing data is a limitation of the study and we acknowledge that results in our multivariate analysis suffer to these missing data. In the multivariate analysis we have handled missing values as independent variable. As OR related to missing values are very high, this result in a conservative OR estimate for patients with registered abnormal vital values closer to one than we believe it would have been if no data were missing.

We have not taken do-not-attempt-to-resuscitate orders or terminal diagnoses into account. These patients are often admitted to palliative care until death or discharged to home after initial assessment. They are also a group of patients with many missing data in vital values. However, these patients are always initially triaged including registration of vital values upon arrival to the ED and therefore included in our analysis.

The impact of risk factors does not only vary according to population but also vary over time.

In the overall context, the results concerning risk factors associated with short term mortality following acute ED-admission are inconsistent, and studies differ in population, selection and outcome mortality timespans. Many studies concerning adverse events in the ED are focused on logistic and environmental factors and clinical condition at arrival. The distribution and differences of OR across mortality groups in our study, show that interpretation of studies conducted in emergency settings, should take outcome mortality measures into account, when comparing studies on risk factors and developing risk prognostication models. 


\section{Strength and limitations}

The main strength of our study is the cohort, consisting of consecutive patients registered in the ED during the study period. All patients had complete follow-up data, combined with data from national, validated registries, which are highly complete. The present single-center study focused on an ED at a University Hospital and interpretations may not be generalizable to other settings. However, our study cohort comprised of broad typical ED population, with patients entering the ED, due to medical or surgical conditions. Thus, our results reflect a highly diverse patient group.

In theory, all ED visits in Denmark are preceded by contact with a physician from primary sector, thereby avoiding unnecessary contacts to the ED. The proportion of events are, however, probably similar to other ED's with similar logistic and regional setup, since patients eventually admitted are probably alike [27].

We chose to use ED-discharge-diagnoses and not hospital-discharge-diagnoses, since complications to the primary condition might later occur and cause registration of different discharge diagnoses.

Missing data is always a limitation to a study and the proportion of missing in databases of acute settings are well-known. Several research groups have evaluated the effects of missing data on analysis from trauma databases and different techniques for handling missing data $[28,29]$. In our study, missing data in vital values may represent immeasurable low or high values, normal values observed but not registered, values of zero or simple lack of registration due to other circumstances surrounding the patient. We have chosen to handle missing data as an independent variable in the multivariable analysis and find that the association with mortality demonstrates that missing data is not missing at random.

The reporting of this study conforms to the STROBE statement [30].

\section{Conclusion}

Among adult patients registered alive at arrival in the ED, we showed an over-all 30 -day mortality of $4 \%$. Approximately one third dies within 2 days after arrival to the ED. Strong risk factors for immediate mortality are abnormal vital values and a high degree of acuity at arrival to the ED. These risk factors declined considerable according to increasing time from arrival till death. In contrast increasing age, male sex and increasing comorbidity were all mortality risk factors, but their odds ratios did not diminish according to time from arrival to death (Tables 2 and 3).

\section{Additional file}

Additional file 1: DEPT triage. Vital values. (XLSX $61.8 \mathrm{~kb})$

\section{Abbreviations}

95\%Cl: 95\% confidence interval; ED: Emergency Department; MECU: Mobile Emergency Care Unit; OR: Odds ratio; OUH: Odense University Hospital

\section{Acknowledgements}

We owe our data manager Niels Ibsgaard Agerbek a sincere thank you for assisting with data extraction and management.

\section{Funding}

CNB is financially supported by PhD salary grants from the Danish National Innovation Foundation, The University of Southern Denmark and from Odense University Hospital. AL is supported by an unrestricted grant given to University of Southern Denmark by the philanthropic fund, The Tryg Foundation.

\section{Availability of data and materials}

In accordance with Danish law, data at person level may not be shared outside of the projects which have permission to use them. We are unable to share person based data in public.

\section{Authors' contributions}

CNB conceived the study including analysis and interpretation of data. CNB drafted the manuscript. AL conceived an coordinated the study and performed critical appraisal of the manuscript. AL, MB and SM all contributed into reviewing and revising the paper and has critically reviewed drafts of the manuscript. All authors edited and approved the final manuscript.

\section{Ethics approval and consent to participate}

In compliance with Danish law, this study was approved by the Danish Data Protection agency (J No 2013-41-2036) and the Danish National Board of Health (J No 3-3013-355). No further ethical approval is needed for registerbased research in Denmark. Data was anonymized immediately after linkage.

\section{Consent for publication}

The study is registered with and approved by the Danish Data Protection Agency (J NO 2013-41-2036). Access to patient records is approved by the Danish Health and Medicines Authority (J NO 3-30013-355/1).

\section{Competing interests}

The authors declare that they have no competing interest.

\section{Publisher's Note}

Springer Nature remains neutral with regard to jurisdictional claims in published maps and institutional affiliations.

\section{Author details}

${ }^{1}$ Department of Emergency Medicine, Odense University Hospital, Sdr. Boulevard 29, entrance 130, 1st floor, DK-5000 Odense C, Denmark. ${ }^{2}$ Department of Emergency Medicine, University of Southern Denmark, Odense University Hospital, Odense, Denmark. ${ }^{3}$ Mobile Emergency Care Unit, Department of Anaesthesiology and Intensive Care Medicine, Odense University Hospital, Odense, Denmark. ${ }^{4}$ Department of Emergency Medicine, Odense University Hospital, Odense, Denmark.

Received: 22 August 2017 Accepted: 27 March 2018

Published online: 20 April 2018

\section{References}

1. Kause J, et al. A comparison of antecedents to cardiac arrests, deaths and emergency intensive care admissions in Australia and New Zealand, and the United Kingdom-the ACADEMIA study. Resuscitation. 2004;62(3):275-82.

2. Buist $M$, et al. Association between clinically abnormal observations and subsequent in-hospital mortality: a prospective study. Resuscitation. 2004; 62(2):137-41.

3. Jacques $T$, et al. Signs of critical conditions and emergency responses (SOCCER): a model for predicting adverse events in the inpatient setting. Resuscitation. 2006;69(2):175-83.

4. Merz TM, et al. Risk assessment in the first fifteen minutes: a prospective cohort study of a simple physiological scoring system in the emergency department. Crit Care. 2011;15(1):R25. 
5. Finkelsztein EJ, et al. Comparison of qSOFA and SIRS for predicting adverse outcomes of patients with suspicion of sepsis outside the intensive care unit. Crit Care. 2017;21(1):73.

6. Singer AJ, et al. Quick SOFA scores predict mortality in adult emergency department patients with and without suspected infection. Ann Emerg Med. 2017;69(4):475-9.

7. Freemantle $\mathrm{N}$, et al. Weekend hospitalization and additional risk of death: an analysis of inpatient data. J R Soc Med. 2012;105(2):74-84.

8. Aylin P, et al. Weekend mortality for emergency admissions. A large, multicentre study. Qual Saf Health Care. 2010;19(3):213-7.

9. Concha OP, et al. Do variations in hospital mortality patterns after weekend admission reflect reduced quality of care or different patient cohorts? A population-based study. BMJ Qual Saf. 2014;23(3):215-22.

10. Shiue I, McMeekin P, Price C. Retrospective observational study of emergency admission, readmission and the 'weekend effect'. BMJ Open. 2017;7(3):e012493.

11. Shih YN, et al. Association of weekend effect with early mortality in severe sepsis patients over time. J Inf Secur. 2017;74(4):345-51.

12. Vest-Hansen B, et al. Out-of-hours and weekend admissions to Danish medical departments: admission rates and 30-day mortality for 20 common medical conditions. BMJ Open. 2015;5(3):e006731.

13. Handel $A E$, et al. Weekend admissions as an independent predictor of mortality: an analysis of Scottish hospital admissions. BMJ Open. 2012:2(6).

14. Noad R, Stevenson M, Herity NA. Analysis of weekend effect on 30-day mortality among patients with acute myocardial infarction. Open Heart. 2017:4(1)::000504

15. Churpek MM, et al. Quick Sepsis-related organ failure assessment, systemic inflammatory response syndrome, and early warning scores for detecting clinical deterioration in infected patients outside the intensive care unit. Am J Respir Crit Care Med. 2017;195(7):906-11.

16. Sager $R$, et al. Are admission procalcitonin levels universal mortality predictors across different medical emergency patient populations? Results from the multi-national, prospective, observational TRIAGE study. Clin Chem Lab Med. 2017;55(12).

17. Singer $\mathrm{M}$, et al. The third international consensus definitions for Sepsis and septic shock (Sepsis-3). JAMA. 2016;315(8):801-10.

18. Mikkelsen $\mathrm{S}$, et al. Characteristics and prognoses of patients treated by an anaesthesiologist-manned prehospital emergency care unit. A retrospective cohort study. BMJ Open. 2017;7(2):e014383.

19. Schmidt M, Pedersen L, Sorensen HT. The Danish civil registration system as a tool in epidemiology. Eur J Epidemiol. 2014;29(8):541-9.

20. Lynge E, Sandegaard JL, Rebolj M. The Danish National Patient Register. Scand J Public Health. 2011;39(7 Suppl):30-3.

21. Charlson ME, et al. A new method of classifying prognostic comorbidity in longitudinal studies: development and validation. J Chronic Dis. 1987;40(5):373-83.

22. Cretikos $\mathrm{M}$, et al. Guidelines for the uniform reporting of data for medical emergency teams. Resuscitation. 2006;68(1):11-25.

23. Seymour CW, et al. Prehospital systolic blood pressure thresholds: a community-based outcomes study. Acad Emerg Med. 2013;20(6):597-604.

24. Sorensen HT, Sabroe S, Olsen J. A framework for evaluation of secondary data sources for epidemiological research. Int J Epidemiol. 1996;25(2):435-42.

25. Galvagno SM Jr, et al. Association between helicopter vs ground emergency medical services and survival for adults with major trauma. JAMA. 2012; 307(15):1602-10.

26. Stevenson JE, et al. Recording signs of deterioration in acute patients: the documentation of vital signs within electronic health records in patients who suffered in-hospital cardiac arrest. Health Informatics J. 2016;22(1):21-33.

27. Dolton P, Pathania V. Can increased primary care access reduce demand for emergency care? Evidence from England's 7-day GP opening. J Health Econ. 2016;49:193-208

28. Joseph $\mathrm{L}$, et al. Selection bias found in interpreting analyses with missing data for the prehospital index for trauma. J Clin Epidemiol. 2004:57(2):147-53.

29. Sterne JA, et al. Multiple imputation for missing data in epidemiological and clinical research: potential and pitfalls. BMJ. 2009;338:b2393.

30. von Elm E, et al. The strengthening the reporting of observational studies in epidemiology (STROBE) statement: guidelines for reporting observational studies. Int J Surg. 2014;12(12):1495-9.

\section{Submit your next manuscript to BioMed Central and we will help you at every step:}

- We accept pre-submission inquiries

- Our selector tool helps you to find the most relevant journal

- We provide round the clock customer support

- Convenient online submission

- Thorough peer review

- Inclusion in PubMed and all major indexing services

- Maximum visibility for your research

Submit your manuscript at www.biomedcentral.com/submit
Biomed Central 